Bull. Korean Math. Soc. 49 (2012), No. 2, pp. 329-338

http://dx.doi.org/10.4134/BKMS.2012.49.2.329

\title{
H-SLANT SUBMERSIONS
}

\author{
KWANG-SOON PARK
}

\begin{abstract}
In this paper, we define the almost h-slant submersion and the h-slant submersion which may be the extended version of the slant submersion [11]. And then we obtain some theorems which come from the slant submersion's cases. Finally, we construct some examples for the almost h-slant submersions and the h-slant submersions.
\end{abstract}

\section{Introduction}

Given a $C^{\infty}$-submersion $F$ from a Riemannian manifold $\left(M, g_{M}\right)$ onto a Riemannian manifold $\left(N, g_{N}\right)$, there are several kinds of submersions according to the conditions on it: e.g. Riemannian submersion ([5], [10]), slant submersion ([3], [11]), almost Hermitian submersion [12], quaternionic submersion [6], etc. As we know, Riemannian submersions are related with physics and have their applications in the Yang-Mills theory ([2], [13]), Kaluza-Klein theory ([1], [7]), supergravity and superstring theories ([8], [9]), etc. And the quaternionic Kähler manifolds have applications in physics as the target spaces for nonlinear $\sigma$-models with supersymmetry [4]. The paper is organized as follows. In Section 2 we recall some notions needed for this paper. In Section 3 we give the definitions of the almost h-slant submersion and the h-slant submersion and obtain some interesting properties about them. In Section 4 we construct some examples for the almost h-slant submersions and the h-slant submersions.

\section{Preliminaries}

Let $(M, E, g)$ be an almost quaternionic Hermitian manifold, where $M$ is a $4 n$-dimensional differentiable manifold, $g$ is a Riemannian metric on $M$, and $E$ is a rank 3 subbundle of $\operatorname{End}(T M)$ such that for any point $p \in M$ with its some neighborhood $U$, there exists a local basis $\left\{J_{1}, J_{2}, J_{3}\right\}$ of sections of $E$ on $U$ satisfying for all $\alpha \in\{1,2,3\}$

$$
J_{\alpha}^{2}=-i d, \quad J_{\alpha} J_{\alpha+1}=-J_{\alpha+1} J_{\alpha}=J_{\alpha+2},
$$

Received November 2, 2010; Revised May 24, 2011.

2010 Mathematics Subject Classification. 53C15, 53C26, 53C43.

Key words and phrases. Riemannian submersion, Kähler manifold, quaternionic Hermitian manifold, hyperkähler manifold. 


$$
g\left(J_{\alpha} X, J_{\alpha} Y\right)=g(X, Y) \text { for all vector fields } X, Y \text { on } M,
$$

where the indices are taken from $\{1,2,3\}$ modulo 3 . The above basis $\left\{J_{1}, J_{2}, J_{3}\right\}$ is said to be a quaternionic Hermitian basis. We call $(M, E, g)$ a quaternionic Kähler manifold if there exist locally defined 1-forms $\omega_{1}, \omega_{2}, \omega_{3}$ such that for $\alpha \in\{1,2,3\}$

$$
\nabla_{X} J_{\alpha}=\omega_{\alpha+2}(X) J_{\alpha+1}-\omega_{\alpha+1}(X) J_{\alpha+2} \text { for any vector field } X \text { on } M \text {, }
$$

where the indices are taken from $\{1,2,3\}$ modulo 3 . If there exists a global parallel quaternionic Hermitian basis $\left\{J_{1}, J_{2}, J_{3}\right\}$ of sections of $E$ on $M$, then $(M, E, g)$ is said to be hyperkähler. Furthermore, we call $\left(J_{1}, J_{2}, J_{3}, g\right)$ a $h y$ perkähler structure on $M$ and $g$ a hyperkähler metric. Let $\left(M, g_{M}\right)$ and $\left(N, g_{N}\right)$ be Riemannian manifolds and $F: M \mapsto N$ a $C^{\infty}$-submersion. The map $F$ is said to be Riemannian submersion if the differential $F_{*}$ preserves the lengths of horizontal vectors [6]. Let $\left(M, g_{M}, J\right)$ be an almost Hermitian manifold. A Riemannian submersion $F:\left(M, g_{M}, J\right) \mapsto\left(N, g_{N}\right)$ is called a slant submersion if the angle $\theta=\theta(X)$ between $J X$ and the space $\operatorname{ker}\left(F_{*}\right)_{p}$ is constant for any nonzero $X \in T_{p} M$ and $p \in M[11]$. We call $\theta$ a slant angle. For $X \in \Gamma\left(\operatorname{ker} F_{*}\right)$, we have

$$
J X=\phi X+\omega X
$$

where $\phi X$ and $\omega X$ are the vertical and horizontal components of $J X$, respectively. For $Z \in \Gamma\left(\left(\operatorname{ker} F_{*}\right)^{\perp}\right)$, we get

$$
J Z=B Z+C Z,
$$

where $B Z$ and $C Z$ are the vertical and horizontal components of $J Z$, respectively [11]. Let $\left(M, E_{M}, g_{M}\right)$ and $\left(N, E_{N}, g_{N}\right)$ be almost quaternionic Hermitian manifolds. A map $F: M \mapsto N$ is called a $\left(E_{M}, E_{N}\right)$-holomorphic map if given a point $x \in M$, for any $J \in\left(E_{M}\right)_{p}$ there exists $J^{\prime} \in\left(E_{N}\right)_{f(x)}$ such that

$$
F_{*} \circ J=J^{\prime} \circ F_{*} .
$$

A Riemannian submersion $F: M \mapsto N$ which is a $\left(E_{M}, E_{N}\right)$-holomorphic map is called a quaternionic submersion. Moreover, if $\left(M, E_{M}, g_{M}\right)$ is a quaternionic Kähler manifold (or a hyperkähler manifold), then we say that $F$ is a quaternionic Kähler submersion (or a hyperkähler submersion) [6].

Let $\left(M, g_{M}\right)$ and $\left(N, g_{N}\right)$ be Riemannian manifolds and $F:\left(M, g_{M}\right) \mapsto$ $\left(N, g_{N}\right)$ a smooth map. The second fundamental form of $F$ is given by

$$
\left(\nabla F_{*}\right)(X, Y):=\nabla_{F_{*} X} F_{*} Y-F_{*}\left(\nabla_{X} Y\right) \text { for } X, Y \in \Gamma(T M),
$$

where we denote conveniently by $\nabla$ the Levi-Civita connections of the metrics $g_{M}$ and $g_{N}$. Recall that $F$ is said to be harmonic if $\operatorname{trace}\left(\nabla F_{*}\right)=0$ and $F$ is called a totally geodesic map if $\left(\nabla F_{*}\right)(X, Y)=0$ for $X, Y \in \Gamma(T M)$. 


\section{H-slant submersions}

Definition 3.1. Let $\left(M, E, g_{M}\right)$ be an almost quaternionic Hermitian manifold and $\left(N, g_{N}\right)$ a Riemannian manifold. A Riemannian submersion $F$ : $\left(M, E, g_{M}\right) \mapsto\left(N, g_{N}\right)$ is called an almost h-slant submersion if given a point $p \in M$ with its some neighborhood $U$, there exists a quaternionic Hermitian basis $\{I, J, K\}$ of sections of $E$ on $U$ such that for $R \in\{I, J, K\}$ the angle $\theta_{R}=\theta_{R}(X)$ between $R X$ and the space $\operatorname{ker}\left(F_{*}\right)_{q}$ is constant for nonzero $X \in \operatorname{ker}\left(F_{*}\right)_{q}$ and $q \in U$.

We call such a basis $\{I, J, K\}$ an almost h-slant basis.

Definition 3.2. Let $\left(M, E, g_{M}\right)$ be an almost quaternionic Hermitian manifold and $\left(N, g_{N}\right)$ a Riemannian manifold. A Riemannian submersion $F$ : $\left(M, E, g_{M}\right) \mapsto\left(N, g_{N}\right)$ is called a $h$-slant submersion if given a point $p \in M$ with its some neighborhood $U$, there exists a quaternionic Hermitian basis $\{I, J, K\}$ of sections of $E$ on $U$ such that for $R \in\{I, J, K\}$ the angle $\theta_{R}=\theta_{R}(X)$ between $R X$ and the space $\operatorname{ker}\left(F_{*}\right)_{q}$ is constant for nonzero $X \in \operatorname{ker}\left(F_{*}\right)_{q}$ and $q \in U, \theta=\theta_{I}=\theta_{J}=\theta_{K}$.

We call such a basis $\{I, J, K\}$ a $h$-slant basis and the angle $\theta$ h-slant angle. Let $F:\left(M, E, g_{M}\right) \mapsto\left(N, g_{N}\right)$ be an almost h-slant submersion. Then for $X \in \Gamma\left(\operatorname{ker} F_{*}\right)$, we have

$$
R X=\phi_{R} X+\omega_{R} X
$$

where $\phi_{R} X$ and $\omega_{R} X$ are the vertical and horizontal parts of $R X$, respectively, for $R \in\{I, J, K\}$. For $Z \in \Gamma\left(\left(\operatorname{ker} F_{*}\right)^{\perp}\right)$, we get

$$
R Z=B_{R} Z+C_{R} Z,
$$

where $B_{R} Z$ and $C_{R} Z$ are the vertical and horizontal components of $R Z$, respectively, for $R \in\{I, J, K\}$.

Note that we denote the projection morphisms on the distributions ker $F_{*}$ and $\left(\operatorname{ker} F_{*}\right)^{\perp}$ by $\mathcal{V}$ and $\mathcal{H}$, respectively. Define the tensor $\mathcal{T}$ and $\mathcal{A}$ by

$$
\begin{aligned}
\mathcal{A}_{E} F & =\mathcal{H} \nabla_{\mathcal{H} E} \mathcal{V} F+\mathcal{V} \nabla_{\mathcal{H} E} \mathcal{H} F \\
\mathcal{T}_{E} F & =\mathcal{H} \nabla_{\mathcal{V} E} \mathcal{V} F+\mathcal{V} \nabla_{\mathcal{V} E} \mathcal{H} F
\end{aligned}
$$

for vector fields $E, F$ on $M$, where $\nabla$ is the Levi-Civita connection of $g_{M}$.

Theorem 3.1. Let $F$ be an almost h-slant submersion from an almost quaternionic Hermitian manifold $\left(M, E, g_{M}\right)$ onto a Riemannian manifold $\left(N, g_{N}\right)$. Then we get

$$
\phi_{R}^{2} X=-\cos ^{2} \theta_{R} X \quad \text { for } X \in \Gamma\left(\operatorname{ker} F_{*}\right) \text { and } R \in\{I, J, K\},
$$

where $\{I, J, K\}$ is an almost $h$-slant basis with the slant angles $\left\{\theta_{I}, \theta_{J}, \theta_{K}\right\}$.

Proof. By Theorem 3.1 of [11], we have the result. 
Lemma 3.1. Let $F$ be an almost h-slant submersion from a hyperkähler manifold $\left(M, I, J, K, g_{M}\right)$ onto a Riemannian manifold $\left(N, g_{N}\right)$ such that $(I, J, K)$ is an almost $h$-slant basis with the slant angles $\left\{\theta_{I}, \theta_{J}, \theta_{K}\right\}$. If $\omega_{R}$ is parallel, then we have $\mathcal{T}_{\phi_{R} X} \phi_{R} X=-\cos ^{2} \theta_{R} \mathcal{T}_{X} X$ for $X \in \Gamma\left(\operatorname{ker} F_{*}\right)$ and $R \in\{I, J, K\}$.

Proof. By Lemma 3.1 of [11], we get the result.

Lemma 3.2. Let $F$ be a h-slant submersion from a hyperkähler manifold $\left(M, I, J, K, g_{M}\right)$ onto a Riemannian manifold $\left(N, g_{N}\right)$ such that $(I, J, K)$ is $a$ h-slant basis with the h-slant angle $\theta=0$. Then we have

$$
\mathcal{H} \nabla_{R X} R X=-\mathcal{H} \nabla_{X} X \quad \text { and } \quad[R X, X] \in \Gamma\left(\operatorname{ker} F_{*}\right)
$$

for $X \in \Gamma\left(\operatorname{ker} F_{*}\right)$ and $R \in\{I, J, K\}$.

Proof. Since $\theta=0$, by Lemma 3.1, we easily get

$$
\mathcal{H} \nabla_{R X} R X=-\mathcal{H} \nabla_{X} X
$$

for $X \in \Gamma\left(\operatorname{ker} F_{*}\right)$ and $R \in\{I, J, K\}$. For $X \in \Gamma\left(\operatorname{ker} F_{*}\right), Z \in \Gamma\left(\left(\operatorname{ker} F_{*}\right)^{\perp}\right)$, and $R \in\{I, J, K\}$, we have

$$
\begin{aligned}
g_{M}\left(\mathcal{H} \nabla_{R X} R X, Z\right) Z & =-g_{M}\left(\nabla_{R X} X, R Z\right) \\
& =-g_{M}\left(\nabla_{X} R X+[R X, X], R Z\right) \\
& =g_{M}\left(-\mathcal{H} \nabla_{X} X+\mathcal{H} R[R X, X], Z\right)
\end{aligned}
$$

so that we obtain $R[R X, X] \in \Gamma\left(\operatorname{ker} F_{*}\right)$, which implies $[R X, X] \in \Gamma\left(\operatorname{ker} F_{*}\right)$ since $\theta=0$.

Remark 3.1. Let $F$ be a hyperkähler submersion from a hyperkähler manifold $\left(M, I, J, K, g_{M}\right)$ onto an almost quaternionic Hermitian manifold $\left(N, E, g_{N}\right)$. Then it is easy to see the following [6]:

(a) the fibers are hyperkähler manifolds.

(b) the manifold $\left(N, E, g_{N}\right)$ is also hyperkähler.

(c) the map $F$ is a h-slant submersion with the h-slant angle $\theta=0$.

Theorem 3.2. Let $F$ be an almost h-slant submersion from a hyperkähler manifold $\left(M, I, J, K, g_{M}\right)$ onto a Riemannian manifold $\left(N, g_{N}\right)$ such that $(I, J, K)$ is an almost $h$-slant basis. If $\omega_{R}$ is parallel for some $R \in\{I, J, K\}$, then $F$ is a harmonic map.

Proof. We may assume that $\omega_{I}$ is parallel. Since $\left(\nabla F_{*}\right)\left(Z_{1}, Z_{2}\right)=0$ for $Z_{1}, Z_{2} \in$ $\Gamma\left(\left(\operatorname{ker} F_{*}\right)^{\perp}\right)$, we only need to show that $\sum_{i=1}^{2 n}\left(\nabla F_{*}\right)\left(e_{i}, e_{i}\right)=0$, where $\left\{e_{i}\right\}_{i=1}^{2 n}$ is an orthonormal basis of $\operatorname{ker} F_{*}$.

Using Theorem 3.1, we can choose an orthonormal basis $\left\{e_{i}\right\}_{i=1}^{2 n}$ of ker $F_{*}$ such that $e_{2 j}=\sec \theta_{I} \phi_{I} e_{2 j-1}$ for $1 \leq j \leq n$.

Hence,

$$
\sum_{i=1}^{2 n}\left(\nabla F_{*}\right)\left(e_{i}, e_{i}\right)=-\sum_{i=1}^{2 n} F_{*}\left(\mathcal{T}_{e_{i}} e_{i}\right)
$$




$$
=-\sum_{j=1}^{n} F_{*}\left(\mathcal{T}_{e_{2 j-1}} e_{2 j-1}+\mathcal{T}_{\sec \theta_{I} \phi_{I} e_{2 j-1}} \sec \theta_{I} \phi_{I} e_{2 j-1}\right) .
$$

Since $\omega_{I}$ is parallel, by Lemma 3.1, we obtain the result.

Corollary 3.1. Let $F$ be an almost h-slant submersion from a hyperkähler manifold $\left(M, I, J, K, g_{M}\right)$ onto a Riemannian manifold $\left(N, g_{N}\right)$ such that $(I, J$, $K)$ is an almost $h$-slant basis with the slant angles $\left\{\theta_{I}, \theta_{J}, \theta_{K}\right\}$ not all nonzeroes. Then $F$ is a harmonic map.

Proof. We may assume $\theta_{I}=0$. Then it implies $\omega_{I}=0$ so that $\omega_{I}$ is parallel. By Theorem 3.2, we get the result.

Theorem 3.3. Let $F$ be an almost h-slant submersion from a hyperkähler manifold $\left(M, I, J, K, g_{M}\right)$ onto a Riemannian manifold $\left(N, g_{N}\right)$ such that $(I, J, K)$ is an almost $h$-slant basis with the slant angles $\left\{\theta_{I}, \theta_{J}, \theta_{K}\right\}$ all non-zeroes. Then the following conditions are equivalent:

(a) the distribution ker $F_{*}$ defines a totally geodesic foliation on $M$,

(b) $g_{M}\left(\mathcal{H} \nabla_{X} \omega_{I} \phi_{I} Y, Z\right)=g_{M}\left(\mathcal{H} \nabla_{X} \omega_{I} Y, C_{I} Z\right)+g_{M}\left(\mathcal{T}_{X} \omega_{I} Y, B_{I} Z\right)$ for $X, Y \in \Gamma\left(\operatorname{ker} F_{*}\right)$ and $Z \in \Gamma\left(\left(\operatorname{ker} F_{*}\right)^{\perp}\right)$,

(c) $g_{M}\left(\mathcal{H} \nabla_{X} \omega_{J} \phi_{J} Y, Z\right)=g_{M}\left(\mathcal{H} \nabla_{X} \omega_{J} Y, C_{J} Z\right)+g_{M}\left(\mathcal{T}_{X} \omega_{J} Y, B_{J} Z\right)$ for $X, Y \in \Gamma\left(\operatorname{ker} F_{*}\right)$ and $Z \in \Gamma\left(\left(\operatorname{ker} F_{*}\right)^{\perp}\right)$,

(d) $g_{M}\left(\mathcal{H} \nabla_{X} \omega_{K} \phi_{K} Y, Z\right)=g_{M}\left(\mathcal{H} \nabla_{X} \omega_{K} Y, C_{K} Z\right)+g_{M}\left(\mathcal{T}_{X} \omega_{K} Y, B_{K} Z\right)$ for $X, Y \in \Gamma\left(\operatorname{ker} F_{*}\right)$ and $Z \in \Gamma\left(\left(\operatorname{ker} F_{*}\right)^{\perp}\right)$.

Proof. Given a complex structure $R \in\{I, J, K\}$, for $X, Y \in \Gamma\left(\operatorname{ker} F_{*}\right)$ and $Z \in \Gamma\left(\left(\operatorname{ker} F_{*}\right)^{\perp}\right)$, we have

$$
\begin{aligned}
g_{M}\left(\nabla_{X} Y, Z\right)= & g_{M}\left(\nabla_{X} \phi_{R} Y, R Z\right)+g_{M}\left(\nabla_{X} \omega_{R} Y, R Z\right) \\
= & -g_{M}\left(\nabla_{X} \phi_{R}^{2} Y, Z\right)-g_{M}\left(\nabla_{X} \omega_{R} \phi_{R} Y, Z\right) \\
& +g_{M}\left(\nabla_{X} \omega_{R} Y, B_{R} Z\right)+g_{M}\left(\nabla_{X} \omega_{R} Y, C_{R} Z\right) .
\end{aligned}
$$

Using Theorem 3.1, we obtain

$$
\begin{aligned}
g_{M}\left(\nabla_{X} Y, Z\right)= & \cos ^{2} \theta_{R} g_{M}\left(\nabla_{X} Y, Z\right)-g_{M}\left(\mathcal{H} \nabla_{X} \omega_{R} \phi_{R} Y, Z\right) \\
& +g_{M}\left(\mathcal{T}_{X} \omega_{R} Y, B_{R} Z\right)+g_{M}\left(\mathcal{H} \nabla_{X} \omega_{R} Y, C_{R} Z\right)
\end{aligned}
$$

so that

$$
\begin{aligned}
\sin ^{2} \theta_{R} g_{M}\left(\nabla_{X} Y, Z\right)= & -g_{M}\left(\mathcal{H} \nabla_{X} \omega_{R} \phi_{R} Y, Z\right)+g_{M}\left(\mathcal{T}_{X} \omega_{R} Y, B_{R} Z\right) \\
& +g_{M}\left(\mathcal{H} \nabla_{X} \omega_{R} Y, C_{R} Z\right)
\end{aligned}
$$

Hence, we get

$$
(\mathrm{a}) \Leftrightarrow(\mathrm{b}), \quad(\mathrm{a}) \Leftrightarrow(\mathrm{c}) \text {, and }(\mathrm{a}) \Leftrightarrow(\mathrm{d}) .
$$

Therefore, we have the result. 
Theorem 3.4. Let $F$ be an almost h-slant submersion from a hyperkähler manifold $\left(M, I, J, K, g_{M}\right)$ onto a Riemannian manifold $\left(N, g_{N}\right)$ such that $(I, J, K)$ $i s$ an almost $h$-slant basis with the slant angles $\left\{\theta_{I}, \theta_{J}, \theta_{K}\right\}$ all non-zeroes. Then the following conditions are equivalent:

(a) the distribution (ker $\left.F_{*}\right)^{\perp}$ defines a totally geodesic foliation on $M$,

(b) $g_{M}\left(\mathcal{H} \nabla_{Z_{1}} Z_{2}, \omega_{I} \phi_{I} X\right)=g_{M}\left(\mathcal{A}_{Z_{1}} B_{I} Z_{2}+\mathcal{H} \nabla_{Z_{1}} C_{I} Z_{2}, \omega_{I} X\right)$ for $X \in \Gamma\left(\operatorname{ker} F_{*}\right)$ and $Z_{1}, Z_{2} \in \Gamma\left(\left(\operatorname{ker} F_{*}\right)^{\perp}\right)$,

(c) $g_{M}\left(\mathcal{H} \nabla_{Z_{1}} Z_{2}, \omega_{J} \phi_{J} X\right)=g_{M}\left(\mathcal{A}_{Z_{1}} B_{J} Z_{2}+\mathcal{H} \nabla_{Z_{1}} C_{J} Z_{2}, \omega_{J} X\right)$ for $X \in \Gamma\left(\operatorname{ker} F_{*}\right)$ and $Z_{1}, Z_{2} \in \Gamma\left(\left(\operatorname{ker} F_{*}\right)^{\perp}\right)$,

(d) $g_{M}\left(\mathcal{H} \nabla_{Z_{1}} Z_{2}, \omega_{K} \phi_{K} X\right)=g_{M}\left(\mathcal{A}_{Z_{1}} B_{K} Z_{2}+\mathcal{H} \nabla_{Z_{1}} C_{K} Z_{2}, \omega_{K} X\right)$ for $X \in \Gamma\left(\operatorname{ker} F_{*}\right)$ and $Z_{1}, Z_{2} \in \Gamma\left(\left(\operatorname{ker} F_{*}\right)^{\perp}\right)$.

Proof. For $X \in \Gamma\left(\operatorname{ker} F_{*}\right)$ and $Z_{1}, Z_{2} \in \Gamma\left(\left(\operatorname{ker} F_{*}\right)^{\perp}\right)$, we have

$$
\begin{aligned}
g_{M}\left(\nabla_{Z_{1}} Z_{2}, X\right)= & g_{M}\left(\nabla_{Z_{1}}\left(I Z_{2}\right), I X\right) \\
= & g_{M}\left(\nabla_{Z_{1}}\left(I Z_{2}\right), \phi_{I} X\right)+g_{M}\left(\nabla_{Z_{1}}\left(I Z_{2}\right), \omega_{I} X\right) \\
= & \cos ^{2} \theta_{I} \cdot g_{M}\left(\nabla_{Z_{1}} Z_{2}, X\right)-g_{M}\left(\nabla_{Z_{1}} Z_{2}, \omega_{I} \phi_{I} X\right) \\
& +g_{M}\left(\mathcal{A}_{Z_{1}} B_{I} Z_{2}+\mathcal{H} \nabla_{Z_{1}} C_{I} Z_{2}, \omega_{I} X\right)
\end{aligned}
$$

so that

$$
\begin{aligned}
\sin ^{2} \theta_{I} \cdot g_{M}\left(\nabla_{Z_{1}} Z_{2}, X\right)= & -g_{M}\left(\mathcal{H} \nabla_{Z_{1}} Z_{2}, \omega_{I} \phi_{I} X\right) \\
& +g_{M}\left(\mathcal{A}_{Z_{1}} B_{I} Z_{2}+\mathcal{H} \nabla_{Z_{1}} C_{I} Z_{2}, \omega_{I} X\right) .
\end{aligned}
$$

Hence, we get $(\mathrm{a}) \Leftrightarrow(\mathrm{b})$. Similarly, we can obtain $(\mathrm{a}) \Leftrightarrow(\mathrm{c})$ and $(\mathrm{a}) \Leftrightarrow(\mathrm{d})$. Therefore, we get the result.

Theorem 3.5. Let $F$ be an almost h-slant submersion from a hyperkähler manifold $\left(M, I, J, K, g_{M}\right)$ onto a Riemannian manifold $\left(N, g_{N}\right)$ such that $(I, J, K)$ $i s$ an almost $h$-slant basis with the slant angles $\left\{\theta_{I}, \theta_{J}, \theta_{K}\right\}$ all non-zeroes. Then the following conditions are equivalent:

(a) $F$ is totally geodesic,

(b) $g_{M}\left(\mathcal{T}_{X} \omega_{I} Y, B_{I} Z_{1}\right)+g_{M}\left(\mathcal{H} \nabla_{X} \omega_{I} Y, C_{I} Z_{1}\right)=g_{M}\left(\mathcal{H} \nabla_{X} \omega_{I} \phi_{I} Y, Z_{1}\right)$, $g_{M}\left(\mathcal{A}_{Z_{1}} B_{I} Z_{2}+\mathcal{H} \nabla_{Z_{1}} C_{I} Z_{2}, \omega_{I} X\right)=-g_{M}\left(\mathcal{H} \nabla_{Z_{1}} \omega_{I} \phi_{I} X, Z_{2}\right)$ for $X, Y \in \Gamma\left(\operatorname{ker} F_{*}\right)$ and $Z_{1}, Z_{2} \in \Gamma\left(\left(\operatorname{ker} F_{*}\right)^{\perp}\right)$,

(c) $g_{M}\left(\mathcal{T}_{X} \omega_{J} Y, B_{J} Z_{1}\right)+g_{M}\left(\mathcal{H} \nabla_{X} \omega_{J} Y, C_{J} Z_{1}\right)=g_{M}\left(\mathcal{H} \nabla_{X} \omega_{J} \phi_{J} Y, Z_{1}\right)$, $g_{M}\left(\mathcal{A}_{Z_{1}} B_{J} Z_{2}+\mathcal{H} \nabla_{Z_{1}} C_{J} Z_{2}, \omega_{J} X\right)=-g_{M}\left(\mathcal{H} \nabla_{Z_{1}} \omega_{J} \phi_{J} X, Z_{2}\right)$ for $X, Y \in \Gamma\left(\operatorname{ker} F_{*}\right)$ and $Z_{1}, Z_{2} \in \Gamma\left(\left(\operatorname{ker} F_{*}\right)^{\perp}\right)$,

(d) $g_{M}\left(\mathcal{T}_{X} \omega_{K} Y, B_{K} Z_{1}\right)+g_{M}\left(\mathcal{H} \nabla_{X} \omega_{K} Y, C_{K} Z_{1}\right)=g_{M}\left(\mathcal{H} \nabla_{X} \omega_{K} \phi_{K} Y, Z_{1}\right)$, $g_{M}\left(\mathcal{A}_{Z_{1}} B_{K} Z_{2}+\mathcal{H} \nabla_{Z_{1}} C_{K} Z_{2}, \omega_{K} X\right)=-g_{M}\left(\mathcal{H} \nabla_{Z_{1}} \omega_{K} \phi_{K} X, Z_{2}\right)$ for $X, Y \in \Gamma\left(\operatorname{ker} F_{*}\right)$ and $Z_{1}, Z_{2} \in \Gamma\left(\left(\operatorname{ker} F_{*}\right)^{\perp}\right)$.

Proof. Given a complex structure $R \in\{I, J, K\}$, for $X, Y \in \Gamma\left(\operatorname{ker} F_{*}\right)$ and $Z, Z_{1}, Z_{2} \in \Gamma\left(\left(\operatorname{ker} F_{*}\right)^{\perp}\right)$, we have

$$
g_{N}\left(\left(\nabla F_{*}\right)(X, Y), F_{*} Z\right)=g_{M}\left(\nabla_{X} R \phi_{R} Y, Z\right)-g_{M}\left(\nabla_{X} \omega_{R} Y, R Z\right)
$$




$$
\begin{aligned}
= & g_{M}\left(\nabla_{X} \phi_{R}^{2} Y, Z\right)+g_{M}\left(\nabla_{X} \omega_{R} \phi_{R} Y, Z\right) \\
& -g_{M}\left(\nabla_{X} \omega_{R} Y, B_{R} Z\right)-g_{M}\left(\nabla_{X} \omega_{R} Y, C_{R} Z\right) \\
= & -\cos ^{2} \theta_{R} g_{M}\left(\nabla_{X} Y, Z\right)+g_{M}\left(\mathcal{H} \nabla_{X} \omega_{R} \phi_{R} Y, Z\right) \\
& -g_{M}\left(\mathcal{T}_{X} \omega_{R} Y, B_{R} Z\right)-g_{M}\left(\mathcal{H} \nabla_{X} \omega_{R} Y, C_{R} Z\right)
\end{aligned}
$$

so that

$$
\begin{aligned}
\sin ^{2} \theta_{R} g_{N}\left(\left(\nabla F_{*}\right)(X, Y), F_{*} Z\right)= & g_{M}\left(\mathcal{H} \nabla_{X} \omega_{R} \phi_{R} Y, Z\right)-g_{M}\left(\mathcal{T}_{X} \omega_{R} Y, B_{R} Z\right) \\
& -g_{M}\left(\mathcal{H} \nabla_{X} \omega_{R} Y, C_{R} Z\right) .
\end{aligned}
$$

Similarly, we obtain

$$
\begin{aligned}
\sin ^{2} \theta_{R} g_{N}\left(\left(\nabla F_{*}\right)\left(X, Z_{1}\right), F_{*} Z_{2}\right)= & -g_{M}\left(\mathcal{H} \nabla_{Z_{1}} \omega_{R} \phi_{R} X, Z_{2}\right) \\
& -g_{M}\left(\mathcal{A}_{Z_{1}} B_{R} Z_{2}+\mathcal{H} \nabla_{Z_{1}} C_{R} Z_{2}, \omega_{R} X\right) .
\end{aligned}
$$

Hence, we get

$$
\text { (a) } \Leftrightarrow(\mathrm{b}),(\mathrm{a}) \Leftrightarrow(\mathrm{c}) \text {, and }(\mathrm{a}) \Leftrightarrow(\mathrm{d}) .
$$

Therefore, we obtain the result.

Remark 3.2. Let $F$ be a h-slant submersion from a $4 n$-dimensional hyperkähler manifold $\left(M, I, J, K, g_{M}\right)$ onto a $3 n$-dimensional Riemannian manifold $\left(N, g_{N}\right)$ such that $(I, J, K)$ is a h-slant basis with the h-slant angle $\theta=\frac{\pi}{2}$. Since

$$
R\left(\operatorname{ker} F_{*}\right) \perp \operatorname{ker} F_{*} \quad \text { for } R \in\{I, J, K\},
$$

given a local orthonormal frame $\left\{e_{1}, \ldots, e_{n}\right\}$ of ker $F_{*}$, the set $\left\{e_{1}, \ldots, e_{n}, I\left(e_{1}\right)\right.$, $\left.\ldots, I\left(e_{n}\right), J\left(e_{1}\right), \ldots, J\left(e_{n}\right), K\left(e_{1}\right), \ldots, K\left(e_{n}\right)\right\}$ is a local orthonormal frame of $T M$ so that $\left\{I\left(e_{1}\right), \ldots, I\left(e_{n}\right), J\left(e_{1}\right), \ldots, J\left(e_{n}\right), K\left(e_{1}\right), \ldots, K\left(e_{n}\right)\right\}$ is a local orthonormal frame of $\left(\operatorname{ker} F_{*}\right)^{\perp}$.

Let

$$
e_{n+i}:=I\left(e_{i}\right), e_{2 n+i}:=J\left(e_{i}\right), e_{3 n+i}:=K\left(e_{i}\right) \text { for } i \in\{1, \ldots, n\} .
$$

Let

$$
\widetilde{e}_{j}:=F_{*} e_{j} \quad \text { for } n+1 \leq j \leq 4 n .
$$

Since $F$ is a Riemannian submersion, we know

$$
F_{*}([X, Y])=\left[F_{*} X, F_{*} Y\right] \quad \text { and } \quad F_{*}\left(\nabla_{X} Y\right)=\nabla_{F_{*} X} F_{*} Y
$$

for $X, Y \in \Gamma\left(\left(\operatorname{ker} F_{*}\right)^{\perp}\right)$ [11], where we denote conveniently by $\nabla$ the LeviCivita connections of the metrics $g_{M}$ and $g_{N}$.

Assume that

$$
\nabla_{e_{j}} e_{i}=\sum_{k=1}^{4 n} \Gamma_{j i}^{k} e_{k}
$$

for some Christoffel symbols $\Gamma_{j i}^{k}, 1 \leq i, j, k \leq 4 n$.

Clearly,

$$
\Gamma_{j i}^{k}=-\Gamma_{j k}^{i} \quad \text { for } 1 \leq i, j, k \leq 4 n .
$$


Since $\nabla_{e_{j}} R e_{k}=R \nabla_{e_{j}} e_{k}$ for $R \in\{I, J, K\}$ and $j, k \in\{1, \ldots, 4 n\}$, we have

$$
\begin{aligned}
& \Gamma_{j n+k}^{l}=-\Gamma_{j k}^{n+l}, \Gamma_{j n+k}^{n+l}=\Gamma_{j k}^{l}, \Gamma_{j n+k}^{2 n+l}=-\Gamma_{j k}^{3 n+l}, \Gamma_{j n+k}^{3 n+l}=\Gamma_{j k}^{2 n+l}, \\
& \Gamma_{j 2 n+k}^{l}=-\Gamma_{j k}^{2 n+l}, \Gamma_{j 2 n+k}^{n+l}=\Gamma_{j k}^{3 n+l}, \Gamma_{j 2 n+k}^{2 n+l}=\Gamma_{j k}^{l}, \Gamma_{j 2 n+k}^{3 n+l}=-\Gamma_{j k}^{n+l}, \\
& \Gamma_{j 3 n+k}^{l}=-\Gamma_{j k}^{3 n+l}, \Gamma_{j 3 n+k}^{n+l}=-\Gamma_{j k}^{2 n+l}, \Gamma_{j 3 n+k}^{2 n+l}=\Gamma_{j k}^{n+l}, \Gamma_{j 3 n+k}^{3 n+l}=\Gamma_{j k}^{l}
\end{aligned}
$$

for $1 \leq k, l \leq n$ and $1 \leq j \leq 4 n$.

Hence,

$$
\begin{aligned}
\nabla_{\widetilde{e}_{j}} \widetilde{e}_{i}= & F_{*}\left(\nabla_{e_{j}} e_{i}\right) \\
= & \sum_{l=n+1}^{4 n} \Gamma_{j i}^{l} \widetilde{e}_{l} \\
& = \begin{cases}\sum_{l=1}^{n}\left(\Gamma_{j k}^{l} \widetilde{e}_{n+l}-\Gamma_{j k}^{3 n+l} \widetilde{e}_{2 n+l}+\Gamma_{j k}^{2 n+l} \widetilde{e}_{3 n+l}\right), & i=n+k \\
\sum_{l=1}^{n}\left(\Gamma_{j k}^{3 n+l} \widetilde{e}_{n+l}+\Gamma_{j k}^{l} \widetilde{e}_{2 n+l}-\Gamma_{j k}^{n+l} \widetilde{e}_{3 n+l}\right), & i=2 n+k \\
\sum_{l=1}^{n}\left(-\Gamma_{j k}^{2 n+l} \widetilde{e}_{n+l}+\Gamma_{j k}^{n+l} \widetilde{e}_{2 n+l}+\Gamma_{j k}^{l} \widetilde{e}_{3 n+l}\right), & i=3 n+k\end{cases}
\end{aligned}
$$

for $1 \leq k \leq n$.

\section{Examples}

Example 4.1. Define a map $F: \mathbb{R}^{4} \mapsto \mathbb{R}^{3}$ by

$$
F\left(x_{1}, \ldots, x_{4}\right)=\left(x_{1} \sin \alpha-x_{3} \cos \alpha, x_{2}, x_{4}\right) .
$$

Then the map $F$ is a h-slant submersion with the h-slant angle $\theta=\frac{\pi}{2}$.

Example 4.2. Let $F: \mathbb{R}^{4} \mapsto \mathbb{R}^{3}$ be a Riemannian submersion. Then the map $F$ is a h-slant submersion with the h-slant angle $\theta=\frac{\pi}{2}$.

We can check it as follows: Given coordinates $\left(x_{1}, x_{2}, x_{3}, x_{4}\right)$ on $\mathbb{R}^{4}$, we can naturally choose the complex structures $I, J$, and $K$ on $\mathbb{R}^{4}$ defined by

$$
\begin{aligned}
& I\left(\frac{\partial}{\partial x_{1}}\right)=\frac{\partial}{\partial x_{2}}, I\left(\frac{\partial}{\partial x_{2}}\right)=-\frac{\partial}{\partial x_{1}}, I\left(\frac{\partial}{\partial x_{3}}\right)=\frac{\partial}{\partial x_{4}}, I\left(\frac{\partial}{\partial x_{4}}\right)=-\frac{\partial}{\partial x_{3}} \\
& J\left(\frac{\partial}{\partial x_{1}}\right)=\frac{\partial}{\partial x_{3}}, J\left(\frac{\partial}{\partial x_{2}}\right)=-\frac{\partial}{\partial x_{4}}, J\left(\frac{\partial}{\partial x_{3}}\right)=-\frac{\partial}{\partial x_{1}}, J\left(\frac{\partial}{\partial x_{4}}\right)=\frac{\partial}{\partial x_{2}} \\
& K\left(\frac{\partial}{\partial x_{1}}\right)=\frac{\partial}{\partial x_{4}}, K\left(\frac{\partial}{\partial x_{2}}\right)=\frac{\partial}{\partial x_{3}}, K\left(\frac{\partial}{\partial x_{3}}\right)=-\frac{\partial}{\partial x_{2}}, K\left(\frac{\partial}{\partial x_{4}}\right)=-\frac{\partial}{\partial x_{1}} .
\end{aligned}
$$

Since $F$ is a Riemannian submersion, the dimension of the space $\operatorname{ker}\left(F_{*}\right)_{p}$ is equal to 1 for any $p \in \mathbb{R}^{4}$. Using the properties $\langle R X, X\rangle=0$ for $X \in T_{p} \mathbb{R}^{4}$ and $R \in\{I, J, K\}$, where $\langle$,$\rangle denotes the Euclidean metric on \mathbb{R}^{4}$, we have the result. 
Example 4.3. Let $\left(M, I, J, K, g_{M}\right)$ be a $4 n$-dimensional hyperkähler manifold and $\left(N, g_{N}\right)$ a $(4 n-1)$-dimensional Riemannian manifold. Let $F:(M, I, J, K$, $\left.g_{M}\right) \mapsto\left(N, g_{N}\right)$ be a Riemannian submersion. Then the map $F$ is a h-slant submersion with the h-slant angle $\theta=\frac{\pi}{2}$.

Example 4.4. Define a map $F: \mathbb{R}^{8} \mapsto \mathbb{R}^{6}$ by

$$
F\left(x_{1}, \ldots, x_{8}\right)=\left(x_{1} \sin \alpha-x_{3} \cos \alpha, x_{2}, x_{4}, x_{5} \sin \beta-x_{7} \cos \beta, x_{6}, x_{8}\right) .
$$

Then the map $F$ is a h-slant submersion with the h-slant angle $\theta=\frac{\pi}{2}$.

Example 4.5. Let $\left(M_{1}, I_{1}, J_{1}, K_{1}, g_{1}\right)$ be a $4 m$-dimensional hyperkähler manifold and $\left(M_{2}, I_{2}, J_{2}, K_{2}, g_{2}\right) 4 n$-dimensional hyperkähler manifold. Let $\left(N_{1}, g_{1}^{\prime}\right)$ be a $(4 m-1)$-dimensional Riemannian manifold and $\left(N_{2}, g_{2}^{\prime}\right)$ a $(4 n-1)$ dimensional Riemannian manifold. Let $F_{i}:\left(M_{i}, I_{i}, J_{i}, K_{i}, g_{i}\right) \mapsto\left(N_{i}, g_{i}^{\prime}\right)$ be a Riemannian submersion for $i \in\{1,2\}$. Consider the product map $F_{1} \times F_{2}$ : $M_{1} \times M_{2} \mapsto N_{1} \times N_{2}$ given by

$$
\left(F_{1} \times F_{2}\right)(x, y)=\left(F_{1}(x), F_{2}(y)\right) \text { for } x \in M_{1} \text { and } y \in M_{2} \text {. }
$$

Then the map $F_{1} \times F_{2}$ is a h-slant submersion with the h-slant angle $\theta=\frac{\pi}{2}$.

Example 4.6. Let $(M, E, g)$ be an almost quaternionic Hermitian manifold. Let $\pi: T M \mapsto M$ be the natural projection. Then the map $\pi$ is a h-slant submersion with the h-slant angle $\theta=0[6]$.

Example 4.7. Let $\left(M, E_{M}, g_{M}\right)$ and $\left(N, E_{N}, g_{N}\right)$ be almost quaternionic Hermitian manifolds. Let $F: M \mapsto N$ be a quaternionic submersion. Then the map $F$ is a h-slant submersion with the h-slant angle $\theta=0[6]$.

Example 4.8. Define a map $F: \mathbb{R}^{4} \mapsto \mathbb{R}^{2}$ by

$$
F\left(x_{1}, \ldots, x_{4}\right)=\left(\frac{x_{1}}{\sqrt{2}}-\frac{x_{3}}{\sqrt{2}}, \frac{x_{1}}{\sqrt{2}}-\frac{x_{4}}{\sqrt{2}}\right) .
$$

Then the differential $F_{*}$ does not preserves the lengths of horizontal vectors so that $F$ is not a Riemannian submersion. But the map $F$ has the h-slant angle $\theta$ with $\cos \theta=\frac{\sqrt{3}}{3}$.

Example 4.9. Define a map $F: \mathbb{R}^{8} \mapsto \mathbb{R}^{4}$ by

$$
F\left(x_{1}, \ldots, x_{8}\right)=\left(\frac{x_{1}}{\sqrt{2}}-\frac{x_{3}}{\sqrt{2}}, x_{4}, \frac{x_{5}}{\sqrt{2}}-\frac{x_{7}}{\sqrt{2}}, x_{6}\right) .
$$

Then the map $F$ is an almost h-slant submersion with the slant angles $\left\{\frac{\pi}{4}, \frac{\pi}{2}, \frac{\pi}{4}\right\}$.

Example 4.10. Define a map $F: \mathbb{R}^{4} \mapsto \mathbb{R}^{2}$ by

$$
F\left(x_{1}, \ldots, x_{4}\right)=\left(x_{1} \cos \alpha-x_{3} \sin \alpha, x_{2} \sin \beta-x_{4} \cos \beta\right) .
$$

Then the map $F$ is an almost h-slant submersion with the slant angles $\left\{\theta_{I}, \frac{\pi}{2}\right.$, $\left.\theta_{K}\right\}$ such that $\cos \theta_{I}=|\sin (\alpha+\beta)|$ and $\cos \theta_{K}=|\cos (\alpha+\beta)|$. 


\section{References}

[1] J. P. Bourguignon and H. B. Lawson, A mathematician's visit to Kaluza-Klein theory, Rend. Sem. Mat. Univ. Politec. Torino (1989), Special Issue, 143-163.

[2] Stability and isolation phenomena for Yang-Mills fields, Comm. Math. Phys. 79 (1981), no. 2, 189-230.

[3] B. Y. Chen, Geometry of Slant Submanifolds, Katholieke Universiteit Leuven, Louvain, 1990.

[4] V. Cortés, C. Mayer, T. Mohaupt, and F. Saueressig, Special geometry of Euclidean supersymmetry. I. Vector multiplets, J. High Energy Phys. (2004), no. 3, 028, 73 pp.

[5] A. Gray, Pseudo-Riemannian almost product manifolds and submersions, J. Math. Mech. 16 (1967), 715-737.

[6] S. Ianus, R. Mazzocco, and G. E. Vilcu, Riemannian submersions from quaternionic manifolds, Acta Appl. Math. 104 (2008), no. 1, 83-89.

[7] S. Ianus and M. Visinescu, Kaluza-Klein theory with scalar fields and generalised Hopf manifolds, Classical Quantum Gravity 4 (1987), no. 5, 1317-1325.

[8] _ Space-time compactification and Riemannian submersions, The mathematical heritage of C. F. Gauss, 358-371, World Sci. Publ., River Edge, NJ, 1991

[9] M. T. Mustafa, Applications of harmonic morphisms to gravity, J. Math. Phys. 41 (2000), no. 10, 6918-6929.

[10] B. O'Neill, The fundamental equations of a submersion, Michigan Math. J. 13 (1966), 459-469.

[11] B. Sahin, slant submersions from almost Hermitian manifolds, Bull. Math. Soc. Sci. Math. Roumanie Tome 54(102) (2011), no. 1, 93-105.

[12] B. Watson, Almost Hermitian submersions, J. Differential Geometry 11 (1976), no. 1, $147-165$.

[13] — $, G, G^{\prime}$-Riemannian submersions and nonlinear gauge field equations of general relativity, Global analysis-analysis on manifolds, 324-349, Teubner-Texte Math., 57, Teubner, Leipzig, 1983.

Department of Mathematical Sciences

Seoul National University

SEOUL 151-747, KOREA

E-mail address: parkksn@snu.ac.kr 